\title{
Water adsorption enhances electrical conductivity in transparent p-type Cul
}

\author{
Andrea Crovetto, ${ }^{1,}$ a) Hannes Hempel, ${ }^{1}$ Marin Rusu, ${ }^{1}$ Leo Choubrac, ${ }^{1}$ Danny Kojda, ${ }^{2}$ Klaus Habicht, ${ }^{2,3}$ and \\ Thomas Unold ${ }^{1}$ \\ ${ }^{1)}$ Department of Structure and Dynamics of Energy Materials, Helmholtz-Zentrum Berlin für Materialien und Energie, \\ Hahn-Meitner Platz 1, 14109 Berlin, Germany \\ ${ }^{2)}$ Department of Methods for Characterization of Transport Phenomena in Energy Materials, \\ Helmholtz-Zentrum Berlin für Materialien und Energie, Hahn-Meitner Platz 1, 14109 Berlin, \\ Germany \\ ${ }^{3)}$ Institute of Physics and Astronomy, University of Potsdam, Karl-Liebknecht-Str. 24-25, \\ D-14476 Potsdam
}

$\mathrm{CuI}$ has been recently rediscovered as a p-type transparent conductor with a high figure of merit. Even though many metal iodides are hygroscopic, the effect of moisture on the electrical properties of CuI has not been clarified. In this work, we observe a two-fold increase in the conductivity of $\mathrm{CuI}$ after exposure to ambient humidity for 5 hours, followed by slight long-term degradation. Simultaneously, the work function of CuI decreases by almost $1 \mathrm{eV}$, which can explain the large spread in the previously reported work function values. The conductivity increase is partially reversible and is maximized at intermediate humidity levels. Based on the large intra-grain mobility measured by $\mathrm{THz}$ spectroscopy, we suggest that hydration of grain boundaries may be beneficial for the overall hole mobility.

\section{INTRODUCTION}

Electron-doped transparent conductive materials (ntype TCMs) are used routinely as optically transparent electrical contacts in solar cells and displays ${ }^{14}$ The performance of a given thin-film material as a TCM can be quantified using one of the several figures of merit (FOM) proposed by various authors ${ }^{1 / 3} \mathrm{~A}$ widely adopted FOM is simply the ratio between the electrical conductivity and the absorption coefficient of the material averaged over the visible region.$^{3}$ A few different n-type oxides have FOMs between $1 \Omega^{-1}$ and $10 \Omega^{-1}$, which are sufficiently high to satisfy the requirements of many optoelectronic devices. ${ }^{[}$Conversely, the development of hole-doped (p-type) TCMs has proven more difficult due to the deep valence band and high hole effective masses found in many wide band-gap p-type semiconductors. ${ }^{21516}$ As a consequence, the FOM of the current state-of-theart p-type TCMs is more than two orders of magnitude lower than in the best n-type oxides. ${ }^{778}$ If this limitation were overcome, the design options for optoelectronic devices would be greatly expanded and fully transparent electronics could be realized!21516

A promising p-type TCM is the binary compound $\mathrm{CuI}$. Although its transparency and conductivity have been known for over a century 99 it was only recently discovered that growing $\mathrm{CuI}$ by reactive sputtering could enhance its FOM up to the highest values observed for any p-type TCMs ${ }^{7}$ Since these results were achieved without any (intentional) extrinsic doping, CuI has significant potential for further improvement by the controlled incorporation of acceptor impurities such as the Group VI elements $\mathrm{O}, \mathrm{S}$, and $\mathrm{Se}{ }^{10} \mathrm{CuI}$ has several electronic features that can promote a high hole conductivity by

\footnotetext{
a) Electronic mail: andrea.crovetto@helmholtz-berlin.de
}

native doping: an antibonding valence band with strong dispersion due to $\mathrm{Cu}$ d-I p hybridization, 11 a low formation energy for $\mathrm{Cu}$ vacancies, $\frac{12}{,}$ the lack of compensating native dopants, $\frac{13}{,}$ and the clustering of $\mathrm{Cu}$ vacancies into ordered structures that stabilize a Cu-deficient stoichiometry 12

Interestingly, many halide compounds such as $\mathrm{SnI}_{2}$, $\mathrm{PbI}_{2}$, and hybride halide perovksites $\left(\mathrm{CH}_{3} \mathrm{NH}_{3} \mathrm{PbI}_{3}\right.$ and similar) undergo major bulk reactions and structural changes when exposed to moisture ${ }^{14}$ On the other hand, several works in the perovskite and organic solar cell literature refer to $\mathrm{CuI}$ as a hydrophobic and air-stable hole transport layer based on contact angle measurements and its structural stability in air 15 Even though $\mathrm{CuI}$ is structurally stable under ambient conditions, properties of crucial importance for TCM applications (e.g., electrical conductivity and work function) can be sensitive to much smaller amounts of adsorbed water or oxygen than the quantities necessary for bulk reactions. Previous work on stability of the electrical conductivity in $\mathrm{CuI}$ focused only on long time scales (weeks or months)! 7118 In a similar fashion, the $\mathrm{CuI}$ work function reported in several papers was measured after an undefined exposure time to air, without investigating the effect of oxygen or moisture on the work function of a pristine $\mathrm{CuI}$ surface ${ }^{16] 19} 24$ In this work, we investigate changes in the conductivity, work function, and ionization energy of $\mathrm{CuI}$ in the first few hours of exposure to air at different relative humidities $(\mathrm{RH})$.

\section{EXPERIMENTAL DETAILS}

$250 \mathrm{~nm}$-thick $\mathrm{CuI}$ films were grown on fused silica glass by gas-phase iodization of sputter-deposited metallic $\mathrm{Cu}$ films, similar to previous reports $\frac{9 \mid 25}{\text { Iodization }}$ was performed by placing the $\mathrm{Cu}$ films on a hotplate next to iodine pellets, covering both the films and a 


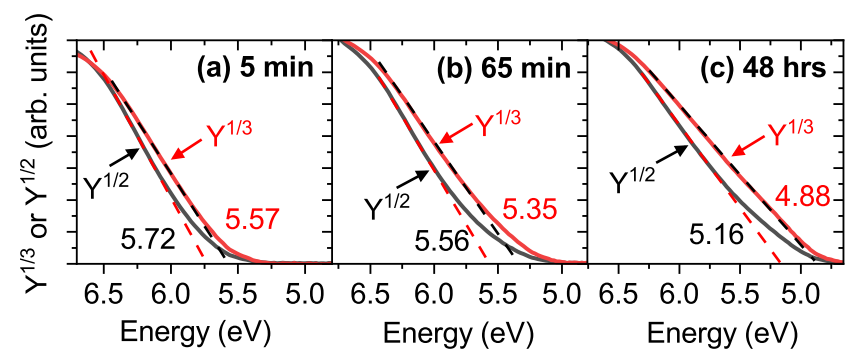

FIG. 1. Determination of the CuI ionization energy by ambient pressure photoemission spectroscopy $5 \mathrm{~min}$ after $\mathrm{CuI}$ film growth (a), 65 min after CuI film growth (b), and $48 \mathrm{~h}$ after $\mathrm{CuI}$ film growth (c). Under the assumption of a nondegenerate (degenerate) semiconductor, the ionization energy can be extracted by linear regression and extrapolation of the $Y^{\frac{1}{3}}$ spectrum ( $Y^{\frac{1}{2}}$ spectrum), where $Y$ is the photoelectron yield ${ }^{26 \mid 27}$ The extrapolated values (in $\mathrm{eV}$ ) are shown. Spectra are normalized.

pellet with an upside-down Petri dish, heating the hotplate to $100^{\circ} \mathrm{C}$ over $2 \mathrm{~min}$, and removing the iodized films from the hotplate 5 min after reaching the temperature setpoint. The films were immediately placed into a nitrogen-filled transport box before characterizing either the electrical conductivity or the surface electronic properties. The time between growth interruption and the first measurement point was 5 min for conductivity measurements and $10 \mathrm{~min}$ for surface measurements. Conductivity measurements were performed with a collinear four-point probe in a humidity-controlled chamber under normal indoor lighting. Work function and ionization energy measurements were performed in the dark using a combined Kelvin probe (KP)/ambient pressure photoemission spectroscopy (PES) system (SKP-5050 and APS-02, KP Technology). Work function and ionization energy were measured sequentially on the same sample as a function of ambient exposure time by using the same tip as a Kelvin probe and as a photoelectron detector. ${ }^{26}$ The ionization energy (Fig. 1) can be determined by linear regression and extrapolation of $Y^{\frac{1}{3}}(E)$ spectra or of $Y^{\frac{1}{2}}(E)$ spectra as shown in Fig. 1. $Y(E)$ is the photoelectron yield versus photon energy $E$, measured by illuminating the sample with monochromated UV light. 26 The $Y^{\frac{1}{3}}$ method is appropriate for nondegenerate semiconductors, ${ }^{27}$ whereas the $Y^{\frac{1}{2}}$ method is appropriate for degenerate semiconductors or metals. ${ }^{26}$ As will be explained later, the Fermi level of the CuI surface changes as a function of ambient exposure time, so both methods are shown in Fig. 1. Work function measurements were calibrated by first measuring the work function of a gold reference sample by PES using the $Y^{\frac{1}{2}}$ method, and by then measuring the contact potential difference (CPD) between the gold reference and the KP tip to determine the tip's work function. The work function of the $\mathrm{CuI}$ films was then calculated by adding the measured CuItip CPD to the work function of the tip.
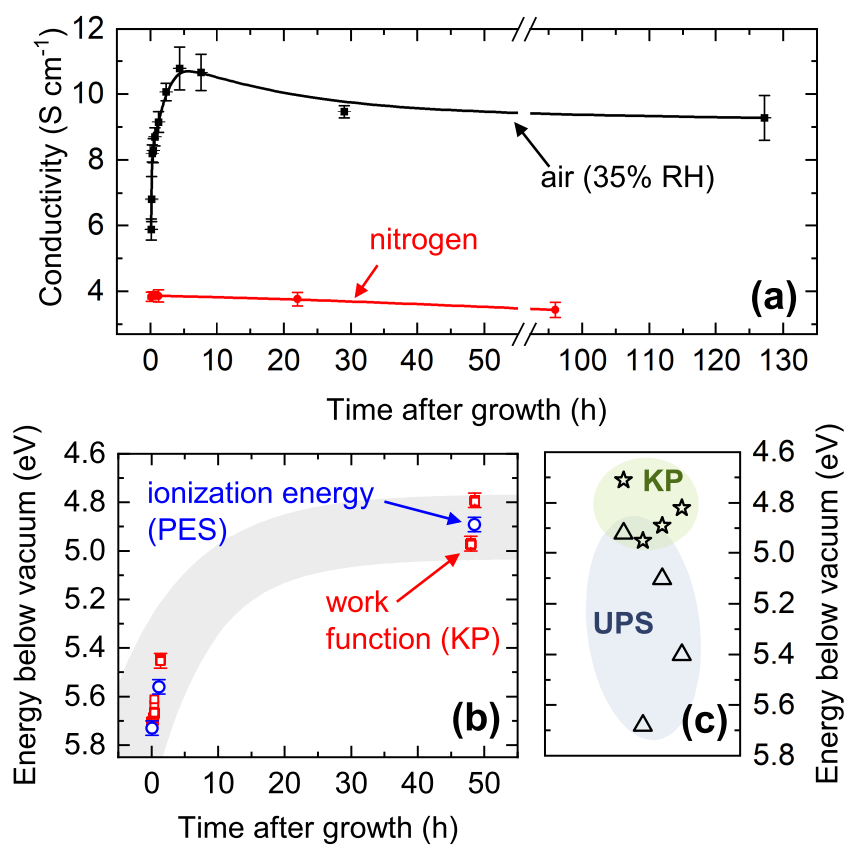

FIG. 2. (a): Conductivity as a function of time for a CuI film stored in air at $35 \% \mathrm{RH}$, and for a nominally identical film stored in a glove box with water content below 1 ppm. (b): Work function (as measured by KP) and ionization energy (as measured by PES) of a CuI film held at $(35 \pm 5) \% \mathrm{RH}$. The shaded region is a guide to the eye. For reasons explained in the main text, the two initial ionization energy data points are determined using the $Y^{\frac{1}{2}}$ method in Fig. 1 (a,b), whereas the final data point is determined using the $Y^{\frac{1}{3}}$ method in Fig. 1. (c). Error bars are $\pm 30 \mathrm{meV}$ for both the ionization energy and the work function. (c): Compilation of work function values measured in previous studies. Star markers are used for KP measurements. ${ }^{19}$ Triangle markers are used for UV photoemission spectroscopy (UPS) measurements. $16|22| 24$

\section{RESULTS AND DISCUSSION}

Basic structural characterization (Figs. S1,S2, Supporting Information) confirms that the $\mathrm{CuI}$ films are in the zincblende phase known for its transparent and conductive properties. Sub-band gap optical transmission is in the $50-80 \%$ range (Fig. S3, Supporting Information). The time evolution of the conductivity for two nominally identical CuI samples is compared in Fig.2(a). One sample was kept at $35 \% \mathrm{RH}$ in the humidity chamber, the other sample was kept in a nitrogen-filled glove box with oxygen and water content below $1 \mathrm{ppm}$. The conductivity of the sample stored in an inert atmosphere slightly decreases over two days. Conversely, the sample stored at ambient humidity experiences a conductivity increase by nearly a factor of two over the first 5 hours after growth. The swift conductivity enhancement is followed by some degradation on a much longer time scale. We verified that the time evolution of the conductivity only depends weakly on the type of background atmosphere (air or ni- 
trogen) but depends strongly on the presence or absence of moisture.

Another nominally identical sample was employed for work function and ionization energy measurements in air with laboratory humidity in the $(35 \pm 5) \% \mathrm{RH}$ range. Similarly to the case of the conductivity, work function and ionization energy undergo rapid changes in the first few hours after growth (Fig. 2(b)). After two days of exposure to ambient humidity, the work function has decreased from $5.70 \mathrm{eV}$ to $4.80 \mathrm{eV}$ and the ionization energy has decreased from $5.57 \mathrm{eV}$ to $4.88 \mathrm{eV}$ using the $Y^{\frac{1}{3}}$ method, or from $5.72 \mathrm{eV}$ to $5.16 \mathrm{eV}$ if the $Y^{\frac{1}{2}}$ method is used (Fig. 1). Work function values reported in the literature ${ }^{16 / 19}$ Z4 are plotted in Fig. 2(c). Comparison to our data suggests that the large spread in the previously measured values may be due to different air exposure times and humidity levels in the previous studies. Consistent with this hypothesis, we note that the reported work functions measured by UV photoemission spectroscopy in ultra-high vacuum are generally higher than those measured by Kelvin probe (Fig. 2(c)), which could be due to (partial) water desorption in a vacuum environment. The work function change $\Delta \Phi$ is caused by preferential alignment of the dipole moments of adsorbed water molecules, which generates a net surface dipole. Modeling the water dipole layer as a plane capacitor yields

$$
\frac{\Delta \Phi}{e}=\frac{n \mu_{\perp} d}{\varepsilon_{0} \varepsilon_{\mathrm{r}}}
$$

In Eq. 1, $n \simeq 9.2 \mathrm{~nm}^{-2}$ is the area density of one monolayer (ML) of adsorbed water molecules, assuming the equilibrium intermolecular separation of $3.55 \AA$ and hexagonal close packing. ${ }^{28} \mu_{\perp}$ is the surface-normal component of the vector average of the dipole moments of all water molecules (Fig. 3(a)). $d$ is the number of adsorbed MLs at $35 \% \mathrm{RH}$, which according to the BET theory $\sqrt{29}$ is between 0.5 ML (strongly hydrophobic surface) and 1.5 ML (strongly hydrophilic surface). Since contact angle measurements indicate that the $\mathrm{CuI}$ surface is an intermediate case $\frac{15 \mid 16}{\sqrt{16}}$ we assume $d=1 \mathrm{ML}$. $\varepsilon_{0}$ and $\varepsilon_{\mathrm{r}}$ are the permittivities of vacuum and of the water layer respectively. We take $\varepsilon_{\mathrm{r}}=1$ because the water molecules generate the dipole field themselves, instead of responding to it as a dielectric. Furthermore, the first ML of adsorbed water is known to be immobile, with $\varepsilon_{\mathrm{r}} \simeq 1$ measured under an externally applied voltage $\frac{30}{30}$ in stark contrast to $\varepsilon_{\mathrm{r}} \simeq 80$ in bulk water. Taking $\Delta \Phi=-0.90 \mathrm{eV}$ as determined by $\mathrm{KP}$ after two days of ambient exposure, we find that $\mu_{\perp}=0.14 \mu_{\mathrm{H}_{2} \mathrm{O}}$, where $\mu_{\mathrm{H}_{2} \mathrm{O}}$ is the magnitude of the dipole moment of a single water molecule $(1.85 \mathrm{D})$. Assuming that a close-packed monolayer of adsorbed water has formed on $\mathrm{CuI}$, the surface dipole has therefore around $14 \%$ of the maximum dipole strength of a perfectly aligned water monolayer. Since the work function decreases upon water adsorption, $\vec{\mu}_{\perp}$ points away from the CuI film. Therefore adsorbed water is preferentially bonded to the CuI surface through its oxygen atoms (Fig. 3(a)), as systematically observed

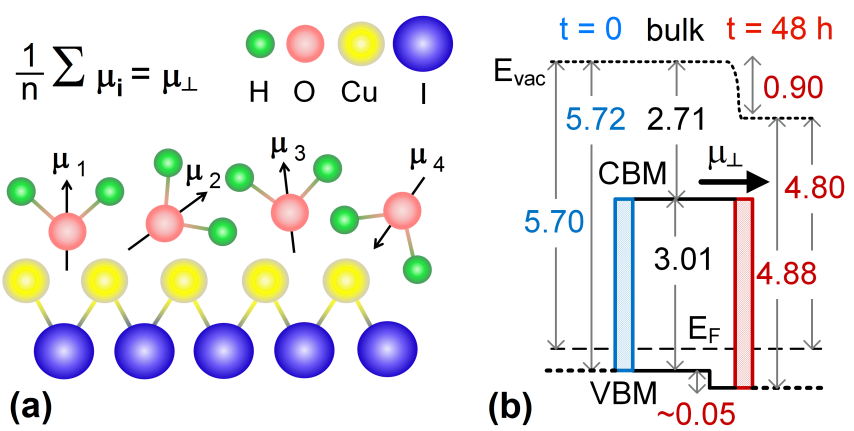

FIG. 3. (a) Simplified sketch of the mechanism generating a surface dipole by water adsorption on CuI. The average perpendicular component of the dipole moment of a water molecule $\left(\mu_{\perp}\right)$ is given by the vector average of the individual water dipoles $\vec{\mu}_{\mathrm{i}}$. The surface is drawn as $\mathrm{Cu}$-terminated due to the high volatility of I. (b) Band diagram of a CuI surface shortly after growth $(t=0$, left side) and after exposure to ambient humidity for 48 hours ( $t=48 \mathrm{~h}$, right side). VBM and CBM are the valence band maximum and conduction band minimum respectively, and $E_{\mathrm{vac}}$ is the vacuum level. Ionization energy and work function data are taken from Fig. 2(b). The band gap $E_{\mathrm{g}}$ is estimated as $3.01 \mathrm{eV}$ using a Tauc plot for direct gap materials (Fig. S4, Supporting Information). Based on this band gap value, the electron affinity of the as-grown surface $(2.71 \mathrm{eV})$ is derived.

in many oxides, chalcogenides, and pnictides. ${ }^{31 / 32}$

Two features of the plot in Fig. 2(b) require further discussion. The first is the negative shift in the measured work function just before and just after performing a PES measurement. The shift is negligible on the as-grown surface but it becomes larger for increasing ambient exposure time. A substantial shift of $-200 \mathrm{meV}$ is observed 48 hours after growth, as shown in detail in Fig. S5(c), Supporting Information. This effect can be explained by photoinduced hydrophilicity, a well-known phenomenon in, e.g., various metal oxides and graphene ${ }^{33134}$ Briefly, the UV radiation used during PES measurements can promote surface hydroxylation by dissociating adsorbed water molecules ${ }^{34}$ or cause surface structural changes that enhance water adsorption. ${ }^{33}$ These effects can increase $n, d$, or $\mu_{\perp}$ in Eq. 11 and thus shift the work function to even lower values. However, photoinduced work function changes are at least partially reversible, since the work function slowly increases again with time once UV irradiation is turned off (Fig. S5(c), Supporting Information).

A second issue is the applicability of the $Y^{\frac{1}{3}}$ method versus the $Y^{\frac{1}{2}}$ method to determine the ionization energy of CuI. In the initial stages of water adsorption, the $Y^{\frac{1}{3}}$ method yields an ionization energy that is $130 \mathrm{meV}$ smaller than the work function, implying very high degenerate p-type doping at the surface of the as-grown film. As the $Y^{\frac{1}{3}}$ method is only valid for nondegenerate semiconductors, we conclude that the $Y^{\frac{1}{2}}$ method for degenerate semiconductors is the most appropriate in the initial stages of ambient exposure. In fact, the $Y^{\frac{1}{2}}$ method yields an ionization energy of $5.72 \mathrm{eV}$ on the 

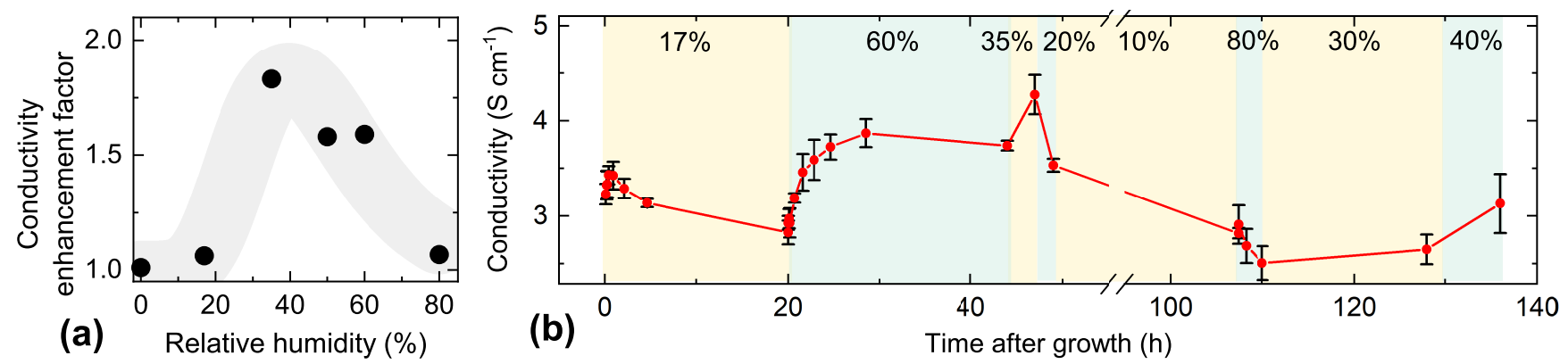

FIG. 4. (a): Conductivity enhancement factor (maximum conductivity divided by initial conductivity) as a function of relative humidity. Each data point refers to a unique $\mathrm{CuI}$ film only exposed to a single humidity. The shaded region is a guide to the eye. (b): Conductivity trace of a single CuI sample sequentially exposed to different humidities.

as-grown surface, which is $20 \mathrm{meV}$ larger than the work function. This value is in good agreement with (bulk) thermovoltage measurements, which yield a bulk doping density of $(2 \pm 1) \times 10^{18} \mathrm{~cm}^{-3}$, thus implying a Fermi level $(2 \pm 27) \mathrm{meV}$ above the valence band maximum (VBM) in the CuI bulk using Fermi statistics. A surface band diagram based on these values is sketched on the left side of Fig. 3(b), and labeled as " $t=0$ " to represent the as-grown surface. Since the Fermi levels on the asgrown surface and in the bulk are found to coincide, the $\mathrm{CuI}$ bands and the vacuum level are drawn flat. On the other hand, applying the $Y^{\frac{1}{2}}$ method after $48 \mathrm{~h}$ of ambient exposure yields an ionization energy that is $360 \mathrm{meV}$ larger than the work function (compare Fig. 1(c) and Fig. 2(b)) significantly outside the applicability range of the method. Hence, the $Y^{\frac{1}{3}}$ method for nondegenerate semiconductors should be employed at this later stage. The corresponding ionization energy is $4.88 \mathrm{eV}$, which is $80 \mathrm{meV}$ lower than the work function, thus confirming the applicability of the $Y^{\frac{1}{3}}$ method at this stage. A surface band diagram for the ambient-exposed CuI surface is shown on the right side of Fig. 3(b) and is labeled as " $t=24 \mathrm{~h}$ ". The surface dipole, given by $\Delta \Phi=-0.90 \mathrm{eV}$, is represented by the abrupt shift in the vacuum level $E_{\mathrm{vac}}$. Since the ionization energy decreases slightly less than the work function from $t=0$ to $t=24 \mathrm{~h}(-0.84 \mathrm{eV}$ versus $-0.90 \mathrm{eV}$ ), we do not exclude the possibility of a small VBM downshift at the ambient-exposed surface due to chemical effects such as, e.g., surface oxidation.

To investigate the origin of the conductivity enhancement upon water adsorption, the time-dependent conductivity experiment shown in Fig. 2(a) was repeated at six different RHs between 0 and $80 \%$, with a fresh sample grown before each new experiment. At each $\mathrm{RH}$, we plot the ratio between the maximum conductivity over time and the initial conductivity of the asgrown surface (Fig. 4(a)). This conductivity enhancement factor is maximized when $\mathrm{RH}$ is around $30-40 \%$ and is negligible at very low and very high humidities. Additional CuI samples with a different thickness confirm this trend (Fig. S6, Supporting Information). Simply plotting the maximum conductivity over time versus
$\mathrm{RH}$ also results in a similar trend (Fig. S7, Supporting Information). Since a RH of $30-40 \%$ corresponds to about $1 \mathrm{ML}$ coverage as explained above, we conclude that the conductivity-enhancing mechanism is maximized by the presence of no more than 1 ML of adsorbed water. Furthermore, the time evolution of conductivity and work function are similar to each other (Figs. 2(a,b)) with a fast initial transient in the first couple of hours after exposure to moist air. These findings provide strong evidence against bulk effects, such as increased hole doping in $\mathrm{CuI}$ by incorporation of, e.g., $\mathrm{O}_{\mathrm{I}}$ acceptors in the bulk. Instead, the conductivity enhancement seems to be related directly to the decrease in work function at $\mathrm{CuI}$ surfaces, although it cannot simply be attributed to a decrease in contact resistance as the four-point measurement configuration excludes contributions from contact resistance. Another option is the existence of a parallel conduction path through the adsorbed water layer at the film surface, which could lead to an increase in the measured conductivity. However, the conductivity of adsorbed water is not higher than a few $\mathrm{mS} / \mathrm{cm}$ even under extreme humidity $[35$ As the conductivity of our as-grown $\mathrm{CuI}$ is about three orders of magnitude higher (Fig. 2(a)), the adsorbed water layer cannot be regarded as a high-conductivity path. Surface doping effects can also be excluded because the Fermi level of the humidity-exposed surface (right side of Fig. 3(b)) is further away from the VBM compared to the Fermi level of the as-grown surface (left side of Fig. 3(b)), indicating a lower surface hole concentration after water adsorption.

Instead, we propose the following explanation for the moisture-enhanced conductivity. The as-grown surface is highly doped (Fig. 3(b)) but highly defective due to unpassivated surface dangling bonds. Once a ML of water has been adsorbed, the surface becomes more weakly doped (Fig. 3(b)) but the surface dangling bonds become saturated by water molecules. Since the grain boundaries of various polycrystalline iodides are known to experience fast hydration in a humid environment $\frac{14 / 36}{14}$ we assume that the $\mathrm{CuI}$ grain boundaries become hydrated in a similar time frame as the film surface characterized in this work. The phenomena discussed above are unlikely 


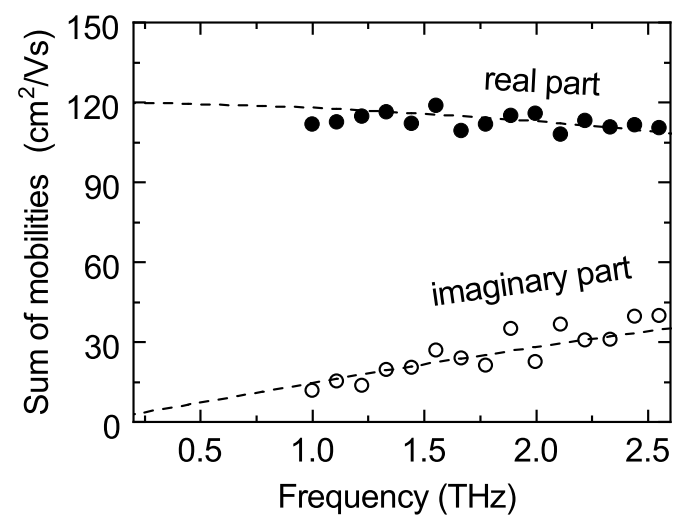

FIG. 5. Sum $\Sigma_{\mu}$ of electron- and hole mobility in a CuI film deposited on fused silica glass, as measured by $\mathrm{THz}$ transmission spectroscopy using a $400 \mathrm{~nm}$ laser pump to generate free carriers. The dashed lines are fits to the real and imaginary parts of $\Sigma_{\mu}$ using the Drude model with a carrier effective mass of $0.3 \mathrm{~m}_{\mathrm{e}}$ and a carrier scattering time of $20 \mathrm{fs}$. The extrapolated value of $\Sigma_{\mu}$ at zero frequency (DC electric field) is $120 \mathrm{~cm}^{2} / \mathrm{Vs}$.

to enhance the hole conductivity along the film surface or grain boundaries due to the lower hole concentration of the humidity-exposed surface. However, it is reasonable to expect enhanced hole transport across grain boundaries because of water passivation of dangling bonds at grain boundaries. Furthermore, the presence of a conductive water layer between grain boundaries may open up more physical transport channels across poorly connected grain boundaries. Despite the presence of a hole barrier due to the water-induced surface dipole (Fig. 3(b)), the thickness of a water ML is only $\sim 1-3 \AA$, so the tunneling probability for holes across grain boundaries is high. If this interpretation of humidity-enhanced conductivity is correct, then grain boundaries act as bottlenecks for hole transport in our $\mathrm{CuI}$ films. Thus, one would expect the intra-grain hole mobility in $\mathrm{CuI}$ to be significantly larger than the long-range hole mobility including transport across various grain boundaries. The former can be measured by $\mathrm{THz}$ spectroscopy ${ }^{37}$ and the latter can be measured using the Hall effect. Indeed, our $\mathrm{THz}$ spectroscopy measurements on $\mathrm{CuI}$ indicate Drude-like conductivity and a value of $120 \mathrm{~cm}^{2} / \mathrm{Vs}$ as the sum of carrier mobilities (Fig. 5). This value is much larger than the hole mobility found by Hall measurements $\left(\sim 6 \mathrm{~cm}^{2} / \mathrm{Vs}\right)$ for films of similar conductivity synthesized in the same way ${ }^{38}$ This discrepancy is compatible with the hypothesis that grain boundaries limit charge transport in $\mathrm{CuI}$ films. This limitation can be mitigated by water adsorption and grain boundary hydration. Interestingly, previous magnetoresistance experiments also attributed the high mobilities of $\mathrm{CuI}$ films to tunneling effects across grain boundaries, although the effect of grain boundary hydration was not considered $[39$ There are no reasons to expect that passivation of $\mathrm{CuI}$ grain boundaries should improve after the first monolayer of water has been ad- sorbed, so the conductivity enhancement factor is not expected to increase at higher humidities, where more than one stable monolayer of water exists. In fact, the conductivity enhancement factor even drops at higher humidities (Fig. 4(a)) due to other unidentified loss mechanisms, such as water absorption in the bulk, or to a too large surface dipole.

Finally, we show in Fig. 4(b) that humidity-dependent conductivity changes have both a reversible and an irreversible component. Unlike the case of Fig. 4(a), in this experiment the same sample was exposed to different humidities sequentially, and its conductivity was monitored. At each RH change, the measured conductivity changes consistently with the data in Fig. 4(a). For example, the conductivity increases when moving from $17 \%$ to $60 \% \mathrm{RH}$ or from $60 \%$ to $35 \% \mathrm{RH}$, and it decreases when moving from $35 \%$ to $20 \% \mathrm{RH}$ or from $20 \%$ to $10 \% \mathrm{RH}$ (Fig. 4(b)). However, there is also a longterm overall trend of decreasing conductivity, as evident when comparing the conductivity at $35 \%$ after $\sim 50 \mathrm{hr}$ and the conductivity at $30-40 \%$ after $\sim 120 \mathrm{hr}$. Slow iodine re-evaporation from the film could be responsible for this effect, since we also observe a slow decrease in conductivity in a $\mathrm{CuI}$ film stored in a water-free atmosphere in nitrogen (Fig. 2(a)). The occurrence of iodine re-evaporation was confirmed by placing a $\mathrm{CuI}$ film next to a $\mathrm{Cu}$ plate in a small sample box and observing coloration of the $\mathrm{Cu}$ plate after a few days of storage in air.

\section{CONCLUSION}

CuI films exposed to moist air experience a conductivity increase and a work function decrease over a similar time frame (a couple of hours). We attribute the conductivity increase to mitigation of grain boundary losses by hole tunneling through water-passivated grain boundaries. The conductivity increase is partially reversible and is maximized at $30-40 \% \mathrm{RH}$, which probably corresponds to one monolayer of adsorbed water. We encourage other researchers to report the atmosphere exposure time and humidity level when presenting $\mathrm{CuI}$ characterization results.

\section{SUPPLEMENTARY INFORMATION}

See supplementary material for structural charaterization by XRD and Raman spectroscopy, band gap determination by optical spectroscopy, additional humiditydependent conductivity experiments, and more detailed plots of the initial and final phases of the conductivity, work function, and ionization energy measurements. 


\section{ACKNOWLEDGEMENTS}

This project has received funding from the European Unions Horizon 2020 research and innovation programme under the Marie Skłodowska-Curie grant agreement No 840751. We acknowledge Lars Steinkopf, Sergiu Levcenko, and José Márquez Prieto for technical assistance and helpful discussions.

${ }^{1}$ K. Ellmer, Nature Photonics 6, 809 (2012).

${ }^{2}$ M. Morales-Masis, S. De Wolf, R. Woods-Robinson, J. W. Ager, and C. Ballif, Advanced Electronic Materials 3, 1600529 (2017)

${ }^{3}$ R. G. Gordon, MRS Bulletin 25, 52 (2000)

${ }^{4}$ A. Crovetto, T. S. Ottsen, E. Stamate, D. Kjær, J. Schou, and O. Hansen, Journal of Physics D: Applied Physics 49, 295101 (2016)

${ }^{5}$ A. N. Fioretti and M. Morales-Masis, Journal of Photonics for Energy 10, $042002(2020)$

${ }^{6}$ H. Kawazoe, M. Yasukawa, H. Hyodo, M. Kurita, H. Yanagi, and H. Hosono, Nature 389, 939 (1997)

${ }^{7}$ C. Yang, M. Knei $\beta$, M. Lorenz, and M. Grundmann, Proceedings

of the National Academy of Sciences 113, 12929 (2016)

${ }^{\gamma}$ R. Woods-Robinson, D. Broberg, A. Faghaninia, A. Jain, S. S. Dwaraknath, and K. A. Persson, Chemistry of Materials 30, 8375 (2018)

${ }^{9}$ K. Bädeker, Annalen der Physik 327, 749 (1907)

${ }^{10}$ M. Graužinyt, S. Botti, M. A. L. Marques, S. Goedecker, and J. A. Flores-Livas, Physical Chemistry Chemical Physics 21, 18839 (2019)

${ }^{11}$ Y. Li, J. Sun, and D. J. Singh, Physical Review Materials 2, 035003 (2018).

${ }^{12}$ S. Jaschik, M. R. G. Marques, M. Seifert, C. Rödl, S. Botti, and M. A. L. Marques, Chemistry of Materials 31, 7877 (2019).

${ }^{13} \mathrm{~J}$. Wang, J. Li, and S.-S. Li, Journal of Applied Physics 110, 054907 (2011).

${ }^{14}$ A. M. A. Leguy, Y. Hu, M. Campoy-Quiles, M. I. Alonso, O. J. Weber, P. Azarhoosh, M. van Schilfgaarde, M. T. Weller, T. Bein, J. Nelson, P. Docampo, and P. R. F. Barnes, Chemistry of Materials 27, 3397 (2015)

${ }^{15}$ S. Shao, J. Liu, J. Zhang, B. Zhang, Z. Xie, Y. Geng, and L. Wang, ACS Applied Materials and Interfaces 4, 5704 (2012)

${ }^{16}$ W. Sun, H. Peng, Y. Li, W. Yan, Z. Liu, Z. Bian, and C. Huang, The Journal of Physical Chemistry C 118, 16806 (2014)

${ }^{17}$ W.-Y. Chen, L.-L. Deng, S.-M. Dai, X. Wang, C.-B. Tian, X.X. Zhan, S.-Y. Xie, R.-B. Huang, and L.-S. Zheng, Journal of Materials Chemistry A 3, 19353 (2015)
${ }^{18}$ V. Raj, T. Lu, M. Lockrey, R. Liu, F. Kremer, L. Li, Y. Liu, H. H. Tan, and C. Jagadish, ACS Applied Materials and Interfaces 11, 24254 (2019)

${ }^{19}$ H. C. Rojas, S. Bellani, F. Fumagalli, G. Tullii, S. Leonardi, M. T. Mayer, M. Schreier, M. Grätzel, G. Lanzani, F. Di Fonzo, and M. R. Antognazza, Energy Environ. Sci. 9, 3710 (2016).

${ }^{20}$ D. K. Kaushik, M. Selvaraj, S. Ramu, and A. Subrahmanyam, Solar Energy Materials and Solar Cells 165, 52 (2017)

${ }^{21}$ S. Das, J.-Y. Choi, and T. Alford, Solar Energy Materials and Solar Cells 133, 255 (2015)

${ }^{22}$ S. Yoon, H. Kim, E.-Y. Shin, Y.-Y. Noh, B. Park, and I. Hwang, physica status solidi (a) 213, 2431 (2016)

${ }^{23}$ S. Shao, J. Liu, J. Zhang, B. Zhang, Z. Xie, Y. Geng, and L. Wang, ACS Applied Materials and Interfaces 4, 5704 (2012).

${ }^{24}$ K. Jeon, H. Jee, M. J. Park, S. Lim, and C. Jeong, Thin Solid Films 660, 613 (2018)

${ }^{25}$ A. Crovetto, A. Hajijafarassar, O. Hansen, B. Seger, I. Chorkendorff, and P. C. K. Vesborg, Chemistry of Materials 32, 3385 (2020)

${ }^{20}$ I. D. Baikie, A. C. Grain, J. Sutherland, and J. Law, Applied Surface Science 323, 45 (2014).

${ }^{27}$ J. R. Harwell, T. K. Baikie, I. D. Baikie, J. L. Payne, C. Ni, J. T. S. Irvine, G. A. Turnbull, and I. D. W. Samuel, Physical Chemistry Chemical Physics 18, 19738 (2016)

${ }^{28}$ E. J. W. Wensink, A. C. Hoffmann, M. E. F. Apol, and H. J. C. Berendsen, Langmuir 16, 7392 (2000)

${ }^{29}$ S. Brunauer, P. H. Emmett, and E. Teller, Journal of the American Chemical Society 60, 309 (1938)

${ }^{30}$ E. McCafferty, V. Pravdic, and A. C. Zettlemoyer, Transactions of the Faraday Society 66, 1720 (1970)

${ }^{31}$ V. Stevanović, S. Lany, D. S. Ginley, W. Tumas, and A. Zunger, Physical Chemistry Chemical Physics 16, 3706 (2014)

${ }^{32}$ T. Mayer, A. Klein, O. Lang, C. Pettenkofer, and W. Jaegermann, Surface Science 269-270, 909 (1992)

${ }^{33}$ M. Miyauchi, A. Nakajima, T. Watanabe, and K. Hashimoto, Chemistry of Materials 14, 2812 (2002)

${ }^{34}$ Z. Xu, Z. Ao, D. Chu, A. Younis, C. M. Li, and S. Li, Scientific Reports 4, 6450 (2015)

${ }^{35}$ R. Guckenberger, M. Heim, G. Cevc, H. Knapp, W. Wiegrabe, and A. Hillebrand, Science 266, 1538 (1994)

${ }^{36}$ J. Bradley Phipps, D. Johnson, and D. Whitmore, Solid State Ionics 5, 393 (1981)

${ }^{3}$ H. Hempel, A. Redinger, I. Repins, C. Moisan, G. Larramona, G. Dennler, M. Handwerg, S. F. Fischer, R. Eichberger, and T. Unold, Journal of Applied Physics 120, 175302 (2016)

${ }^{38}$ F.-L. Schein, H. von Wenckstern, and M. Grundmann, Applied Physics Letters 102, 092109 (2013)

${ }^{39}$ M. Kneiß, C. Yang, J. Barzola-Quiquia, G. Benndorf, H. von Wenckstern, P. Esquinazi, M. Lorenz, and M. Grundmann, Advanced Materials Interfaces 5, 1701411 (2018) 